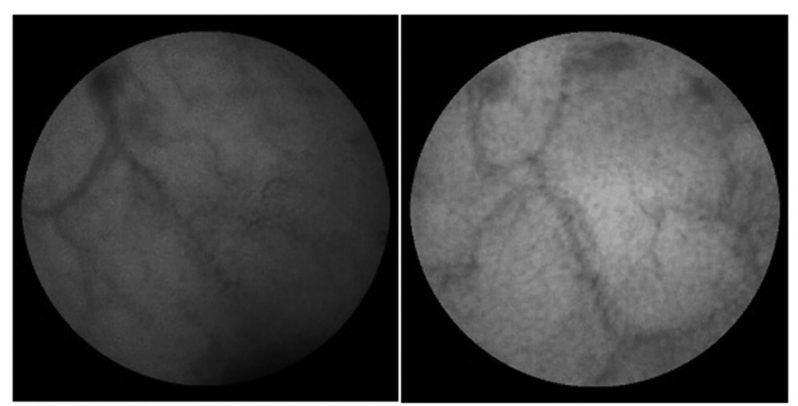

Abstract IDDF2018-ABS-0253 Figure 1 Abnormal (left) and normal (right) visible blood vessels in the small intestine

\section{IDDF2018-ABS-0254 RESPONSE IN SISTER MARY JOSEPH NODE- CAN IT BE A MARKER OF RESPONSE TO THERAPY IN METASTATIC CARCINOMA GALL BLADDER}

Satyabrata Das*. King George's Medical University, India

\subsection{6/gutjnl-2018-IDDFabstracts. 177}

Background Sister Mary Joseph Nodule, a rare presentation of gastrointestinal Malignancy usually signifies a poor prognosis. We present a case of Metastatic carcinoma of the gallbladder which was diagnosed with this unusual presentation. The patient was treated with palliative chemotherapy. Post three cycles of chemotherapy patient showed necrosis in Sister Mary Joseph Nodule. After 6 cycles of palliative chemotherapy patient had stable disease and until seven months of follow up patient was alive.

Methods A 45 year female was diagnosed with metastatic carcinoma gallbladder. On clinical examination, the patient had enlarged gallbladder with an umbellical nodule. FNAC of the Gallbladder mass and umbelllical nodule showed poorly differentiated adenocarcinoma and metastatic adenocarcinoma respectively. The patient was given palliative chemotherapy Gemcitabine on Day1 and Day8. Post 3 such cycles patient was evaluated radiologically and clinically and was found to have stable disease through the umbellical nodule had turned black suggesting necrosis. The patient was given 6 cycles of chemotherapy and then followed every monthly clinically and radiologically.

Results After 7 months of follow up, the patient was alive. There was no recurrence radiologically or clinically and no disease-related Morbidity.
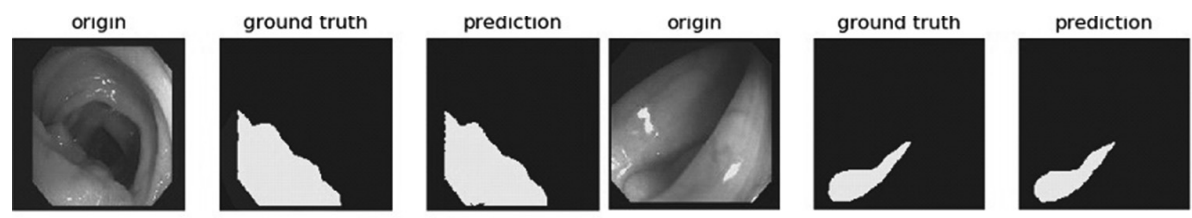

origin
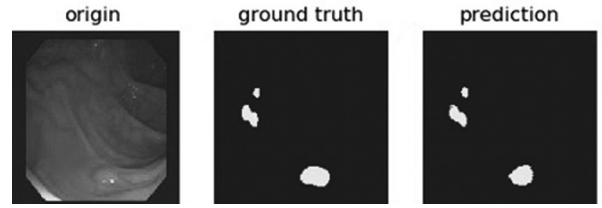

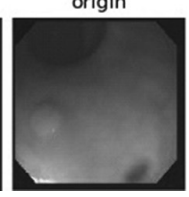

ground truth

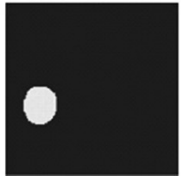

prediction

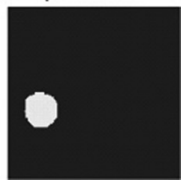

Conclusions A good response to chemotherapy can result in acceptable survival in metastatic carcinoma Gall Bladder. Necrotic change in Sister Mary Joseph Nodule can be a surrogate marker of such response.

\section{IDDF2018-ABS-0257 DETECTING AND SEGMENTING POLYPS USING A DEEP LEARNING-BASED MODEL}

'Liansheng Wang*, 'Shuxin Wang, ${ }^{2}$ Yanxing Hu, 'Shaohui Huang. 'Xiamen University, China; ${ }^{2}$ Xiamen Innovision Medical Technology Company, China

\subsection{6/gutjnl-2018-IDDFabstracts. 178}

Background Colorectal cancer is the third most common cancer in the world, which developed from untreated polyps. Detecting polyps in their early stage have become a serious medical issue. Wireless capsule endoscopy (WCE) was designed to examine the intestinal diseases without surgery and can give a direct visualisation of intestines, in which the time cost is to analyse lots of images to capture the abnormal parts. The doctors will be wearied with about 25000 images per person produced by WCE. This study aims to build a computer-aided diagnosis system to help doctors analyse WCE images and detect polyps.

Methods We use a deep learning-based model to build a computer-aided diagnosis system of WCE images for the assignment of detecting and segmenting polyps. The U-Net network with both dices loss and cross entropy loss is employed in this work, which is considered as an encoding and decoding model. The data used were available from CVC-Colon and CVC-Clinic, it has 992 images with polyps totally. The data is shown in table 1. Comparison to other algorithms, the U-Net model can learn more features automatically and is a high-efficient method for our assignment.

Results The performance of polyp segmentation was evaluated by Dice's coefficient (also known as the Dice coefficient), which presents the degree of similarity between prediction and ground truth. The Dice's coefficient in our study is $84.15 \%$. The segmentation results are shown in IDDF2018-ABS-0257 figure 1 .

Abstract IDDF2018-ABS-0257 Table 1 Data are divided into training data, validation data, and test data

\begin{tabular}{lcccc}
\hline Datasets & $\begin{array}{c}\text { Total } \\
\text { number }\end{array}$ & Training & Validation & Test \\
\end{tabular}

Abstract IDDF2018-ABS-0257 Figure 1 Segmentation results, showing the results of different size and number of polyps 


\begin{tabular}{lllll} 
CVC- & 380 & 300 & 40 & 40 \\
Colon & & & & \\
CVC- & 612 & 492 & 60 & 60 \\
Clinic & & & & \\
\hline
\end{tabular}

Conclusions WCE plays an essential role in diagnosis and prevention of colorectal cancer. However, distinguishing abnormal WCE images with polyps is a significant challenge because it needs high time cost. The results show that our method can help physicians analyse the WCE images and reduce their pressure. This method could be further utilised in the clinical trials to help physicians from the tedious image analysing work.

\section{IDDF2018-ABS-0258 A CASE REPORT OF WARFARIN INDUCED SPONTANEOUS SUB-MUCOSAL AND MESENTERIC HEMATOMA IN THE GASTROINTESTINAL TRACT (SMALL BOWEL) CAUSING BOWEL OBSTRUCTION}

Manuel Jr Velasco*, Mark Anthony De Lusong, Yvonne Mina. Victor R Potenciano Medical Center, Philippines

\subsection{6/gutjnl-2018-IDDFabstracts. 179}

Background To present a rare case of Warfarin-induced spontaneous sub-mucosal and Mesenteric hematoma in the Gastrointestinal Tract (Small Bowel) causing bowel obstruction.

Methods Case Report.

Literature Review.

Results A case of a 67-year-old male is presented with a chief complaint of abdominal pain for three days and difficulty passing stool. He has a known case of Hypertensive atherosclerotic cardiovascular disease, Rheumatic Heart disease, Coronary Artery Disease s/p Coronary Angiography, Chronic Atrial Fibrillation, and Diabetes Mellitus Type II. Maintained on several medications including ASA $80 \mathrm{mg} 1 \mathrm{tab}$ OD and
Coumadin $5 \mathrm{mg} 11 / 2 \mathrm{tab}$ OD. He was lost to follow-up for one year. On his admission, his PT and INR were reportedly undetectable. He was admitted and treated as a case of Small Bowel Ischemia secondary to spontaneous sub-mucosal and Mesenteric haemorrhage and Non- ST Elevation Myocardial Infarction. The entire duration of the hospitalisation took 11 days to include ICU stay, and surgical procedure and recovery. Conclusions This case highlights the risk of haemorrhage in a high-risk patient taking warfarin and the need for close monitoring and follow-up. An intramural hematoma should be entertained in patients on warfarin treatment presenting with clinical signs and symptoms of bowel obstruction. Non-operative treatment of spontaneous small bowel hematoma has a good outcome in most patients. This also stresses the importance of diligent follow-up with the attending physician to ensure a proper balance of anticoagulation and risk of bleeding. On the other hand, this case emphasises that over-anticoagulation leading to bleeding creates negative feedback to the heart, by increasing metabolic demands, which can lead to a paradoxical thromboembolic phenomenon such as Myocardial

\section{IDDF2018-ABS-0259 SEGMENTATION OF INTESTINAL POLYPS VIA A DEEP LEARNING ALGORITHM}

${ }^{1}$ Liansheng Wang*, ${ }^{1}$ Yuqi Qian, ${ }^{2}$ Yanxing Hu. ${ }^{1}$ Xiamen University, China; ${ }^{2}$ Xiamen Innovision Medical Technology Company, China

\subsection{6/gutjnl-2018-IDDFabstracts. 180}

Background Colorectal is the third most commonly occurring cancer in men and the second most commonly occurring cancer in women. At least $80 \%-95 \%$ of colorectal cancers are evolved from intestinal polyps. Therefore early screening of intestinal polyps is one of the crucial ways to prevent colorectal cancer. WCE is widely used in the examination of the internal environment of the digestive tract. However, lots of medical images per patient undoubtedly create a considerable burden on doctors. Thus, in this paper, we describe a
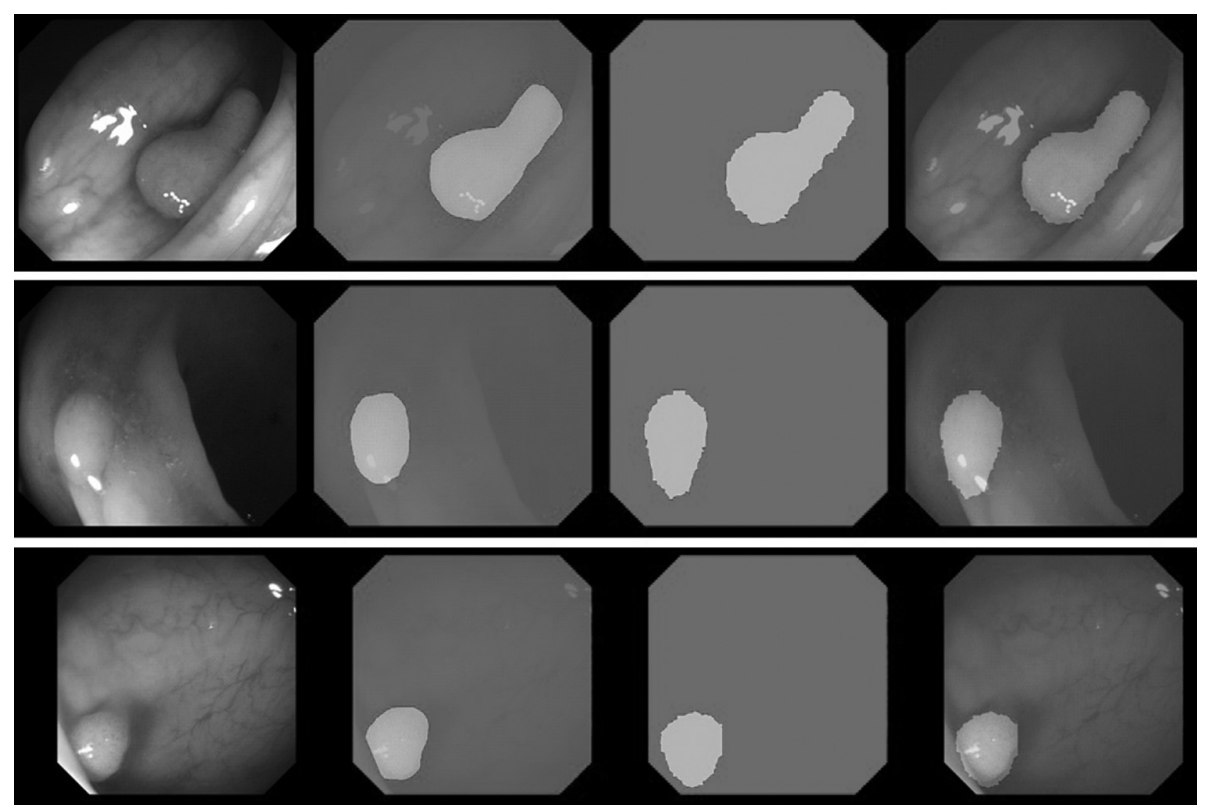

Abstract IDDF2018-ABS-0259 Figure 1 Examples of segmentation results on the test data. Each column represents a single frame, a ground truth annotation, a prediction image, and a prediction image annotation 